\title{
Effect of SSPM Surface Coating on Light Collection Efficiency and Optical Crosstalk for Scintillation Detection
}

\author{
Paul Barton ${ }^{1}$, Christopher Stapels², Erik Johnson², James Christian ${ }^{2}$, Bill Moses ${ }^{3}$, Martin \\ Janecek $^{3}$, David Wehe ${ }^{1}$ \\ ${ }^{1}$ University of Michigan, Ann Arbor, MI ${ }^{2}$ Radiation Monitoring Devices, Inc., Watertown, \\ MA $\quad{ }^{3}$ Lawrence Berkeley National Laboratory, Berkeley, CA
}

A dead region between pixels is required in solid-state photomultipliers (SSPMs) to reduce optical cross-talk to acceptable levels. This reduces the geometric fill factor, which then limits the maximum detection efficiency of the device. By placing an aluminum layer on the silicon wafer above this dead region, scintillation photons that impinge there may be reflected back into the crystal. They may be subsequently redirected towards a sensitive SSPM element. The scintillation photon collection efficiency of the proposed device will be greater than that predicted by the scintillation yield and the ratio of active area alone.

Unfortunately the improved SSPM reflectivity also increases optical crosstalk, since photons emitted during avalanche breakdown may be externally redirected into neighboring pixels. The enhancement of this external optical crosstalk effect was determined through simulation to be of the same order as the improvement in PDE. However, as a function of excess bias, the rate of hot carrier emission will rise much more rapidly than the PDE, setting an upper limit on the applied bias.

Due to spectral mismatch between detection and avalanche emission, the benefits from the gain in PDE will often outweigh the negative effects from additional crosstalk. The spatial dependence of externally-reflected hot carrier emissions was determined to be approximately uniform across the surface of the device. The probabilities of this new optical crosstalk mechanism will be used in conjunction with other previously studied noise mechanisms to form a complete stochastic model of the SSPM.

\section{Motivation}

This work is guided by the derivation of a complete stochastic model of the SSPM. While the first order statistics depend primarily on simple device parameters, the second order statistics are composed of a recursive blend of several non-trivial noise mechanisms. This work is primarily focused on the use of silicon photomultipliers for the readout of scintillating crystals. A crystal in close contact with a photodetector represents a closed optical system in which the photodetector response may depart from that of an open optical system (e.g. laser ranging).

The first difference we consider lies in the ability of the SSPM surface to reflect incoming photons back into the crystal. The aluminum readout lines necessary for interpixel connection may be highly reflective and may also be made to fill the entire dead area between active pixels. The addition of this aluminum fill layer increases the photon detection efficiency of the device by 
giving scintillation photons, which would have been lost in an open optical system, a second chance at detection. The ability of the crystal to return these SSPM-reflected photons back onto the SSPM will depend on the optical properties of the entire system.

\section{Surface Reflectivity}

In a commercial silicon foundry, the exact optical properties of the evaporated aluminum readout lines can vary significantly from process to process. Evaporated aluminum surfaces have been realized with reflectivities of $\sim 94 \%$ at a wavelength of $350 \mathrm{~nm}$.

Radiation Monitoring Devices, Inc. has designed and produced a $3 \mathrm{~mm}$ by $3 \mathrm{~mm}$ four quadrant SSPM (model AE215) with varying pixel width and pitch. An additional aluminum layer adds reflectivity over the entire non-active area. This layer is not perfectly planar due to the readout lines and silicon dioxide below.

Table 1. Geometry of the four quadrants in the RMD AE215 SSPM.

\begin{tabular}{|c|c|c|c|c|c|}
\hline & $\begin{array}{c}\text { Pixel Width } \\
(\mu \mathrm{m})\end{array}$ & $\begin{array}{c}\text { Interpixel } \\
(\mu \mathrm{m})\end{array}$ & $\begin{array}{c}\text { Pixels } \\
\text { Across }\end{array}$ & $\begin{array}{c}\text { Fill Factor } \\
(\%)\end{array}$ & $\begin{array}{c}\text { Al Fill } \\
(\%)\end{array}$ \\
\hline Q1 & 30 & 14 & 31 & 49 & 51 \\
\hline Q2 & 50 & 14 & 21 & 61 & 39 \\
\hline Q3 & 50 & 26 & 18 & 43 & 57 \\
\hline Q4 & 30 & 26 & 24 & 29 & 71 \\
\hline
\end{tabular}

The reflectivity of this SSPM was measured at Lawrence Berkeley National Lab on an apparatus recently designed to measure the reflectance of scintillation crystals and their reflectors. A $400 \mathrm{~nm}$ laser is focused into a one millimeter spot on the surface of the SSPM and an arched bank of photodiodes records the reflected intensity over a solid angle of $2 \pi$. After correcting the measurements for interference effects and the non-planar geometry of the aluminum, the reflectance was estimated to be $\sim 80 \%$ at $400 \mathrm{~nm}$.

\section{Photon Collection Efficiency}

To determine the degree of photon detection efficiency (PDE) improvement from an additional aluminum layer, an optical Monte Carlo simulation was designed using the code DETECT2000 [2]. While the simulation predicts photon collection efficiency, other factors contributing to PDE (e.g. quantum efficiency, avalanche initiation, etc.) are assumed to be constant over the simulation parameters of interest. An object-oriented MATLAB front-end was developed to facilitate model building, geometry and fate plotting, and fast parameterization.

The improvement in collection efficiency was simulated by setting the interpixel area to either perfectly absorbing silicon or $80 \%$ specularly-reflective aluminum. A Teflon-wrapped cubic LYSO crystal $(n=1.825)$ was simulated to be in contact with the SSPM via a thin coupling layer $(\mathrm{n}=1.465)$. In Figure 1, the improvement in photon collection efficiency is observed to be much greater for devices with a smaller fill factor. The defining characteristic of the PDE for a reflective SSPM is its nonlinearity as a function of fill factor. 


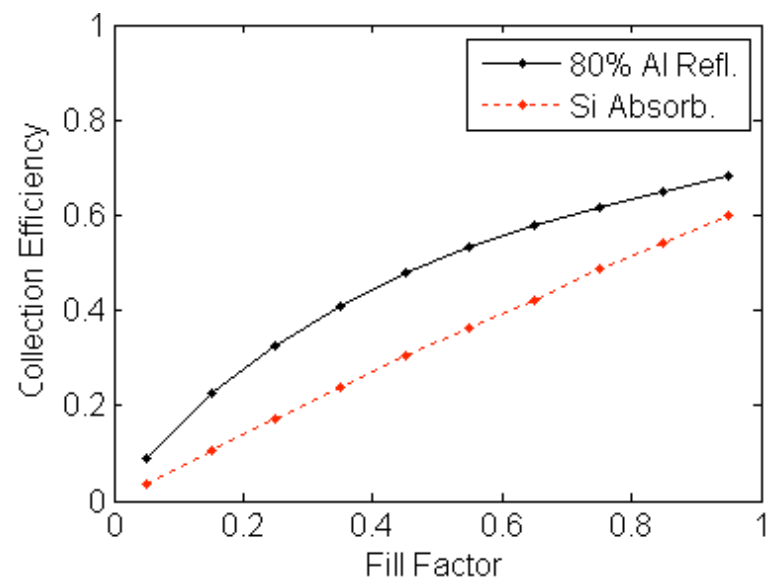

Figure 1. The simulated photon collection efficiency as a function of SSPM fill factor for a Teflon-wrapped cubic crystal on an absorptive (dashed line) or semi-reflective (solid line) detection surface.

Experimental evidence supporting this simulation was difficult to obtain since precise placement of the crystal could only be performed to within $\sim 200 \mu \mathrm{m}$. This represents up to a $30 \%$ difference in detection area between trials. Repeated crystal mounting caused delamination of the SSPM surface, rendering some quadrants unusable. The optical coupling layer (e.g. grease, oil, adhesive, etc.) was also difficult to apply in a manner that would yield consistent optical properties. A viscous coupling layer may also be wicked by capillary action into the Teflon, powder, or other porous crystal reflector, thus altering its optical properties.

\section{Optical Crosstalk Mechanisms}

The optical crosstalk mechanism of the SSPM has often been explained by hot carrier emissions (avalanche photons) traveling laterally through the silicon to a neighboring pixel [3]. Various strategies have been proposed and implemented to reduce this direct optical crosstalk. Recent evidence suggests that a small fraction of avalanche photons may be internally reflected at the bottom silicon interface and initiate subsequent avalanches [4]. We have termed these two mechanisms respectively: internal direct (ID) and internal reflected (IR) optical crosstalk .
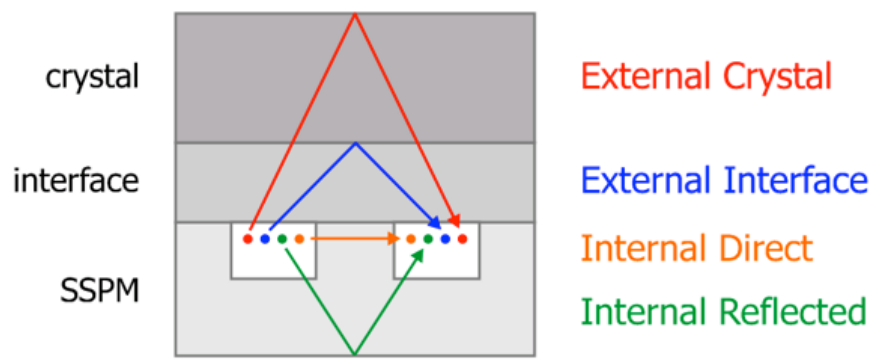

Figure 2. Internal and external optical crosstalk mechanisms in the SSPM as applied in a closed optical system. 
In scintillation spectroscopy, a crystal with a large index of refraction and highly reflective surfaces is mated directly to the SSPM. While ID optical crosstalk can be prevented with proper trench design, the avalanche photons exiting the front face of the detector may strike a crystal reflector and be redirected back towards an active SSPM pixel. Likewise, the requisite optical interface layer may internally reflect avalanche photons based on the mismatch in refractive index with the crystal.

We propose a method for reducing the intensity of avalanche photons while preserving scintillation photons is to make use of their spectral differences. The spectrum of hot carrier emissions is relatively constant in silicon [1,5] and peaks somewhere above $500 \mathrm{~nm}$. Due to the absoprtion coefficient of silicon, the wavelengths that contribute to internal optical crosstalk are primarily confined to 700-1000 $\mathrm{nm}$. However, shorter wavelenght photons are able to exit the surface of the SSPM and are free to return. Therefore, special attention should be paid to the spectral distributions of avalanche photons for internal and external optical crosstalk mechanisms.

Scintillators that peak in the UV-blue may be given priority by application of a suitable optical bandpass filter. For instance, a Schott BG-39 colored glass filter might decrease the transmission of hot carrier emissions more significantly than scintillation photons (Figure 3). The application of this filter may however complicate the system optics and transmission of scintillation photons, negating any potential benefits. Furthermore, a SSPM that is not sensitive to wavelengths higher than say $600 \mathrm{~nm}$ would not benefit from such an application.

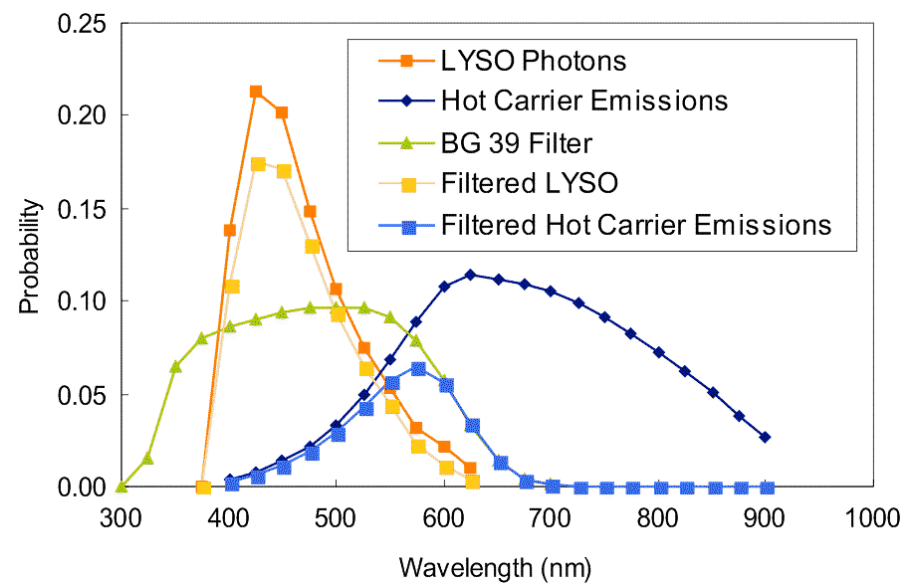

Figure 3. Prediction of emission spectra from LYSO scintillator and silicon avalanche [5] before and after filtering by Schott BG-39 colored glass. Emission spectra are normalized.

The necessity for mitigation of external optical crosstalk photons is greatest for devices with large pixels that are operated at high excess biases and are sensitive to their own hot carrier emissions. The hot carrier emission intensity exiting the face of the SSPM was measured to be exactly proportional to the avalanche current. Reduction of all forms of optical crosstalk can be achieved by reducing pixel size, decreasing quenching times, and otherwise reducing the amount of charge in each avalanche. A blue-sensitive SSPM designed for scintillation detection will also be less sensitive to the longer wavelength hot carrier emissions. 
The avalanche photon surface emission intensity was measured on a PMT for several devices. Those devices with a sharper breakdown voltage transition were observed to exhibit a very fast rise in avalanche emissions. When operating these devices at high excess bias to maximize PDE, small perturbations in bias may lead to large changes in hot carrier emission intensity.

\section{External Optical Crosstalk Simulation}

In support of the objective of simulating the SSPM signal from first principles, a simple distribution is desired from which to sample external optical crosstalk events. This distribution was arrived at by determining the probability that an avalanche photon (hot carrier emission) starting at pixel $i$ interacts with external optical system and lands in pixel $j$. Figure 4a illustrates the simulation geometry for a specularly wrapped cubic LYSO crystal mounted on a 100 pixel SSPM with an intermediate coupling layer. Avalanche photons are emitted isotropically from the surface of each pixel in turn.

a.

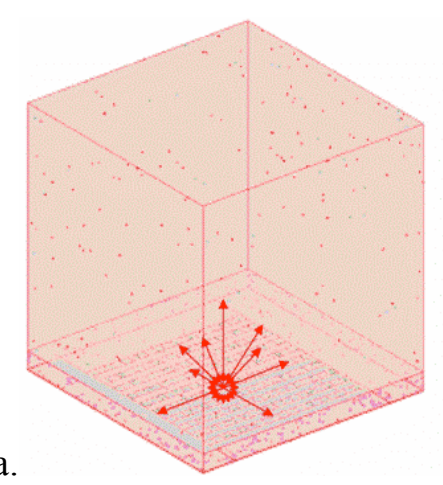

Figure 4. Geometry (a) and response matrix

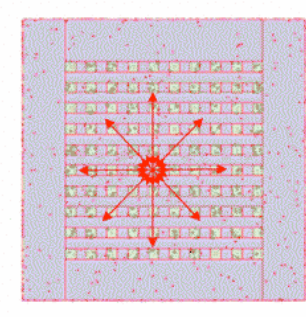

b.

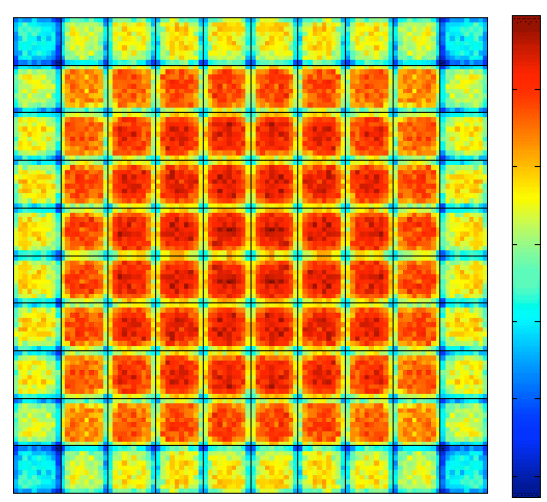

probabilities from a specularly wrapped crystal and reflective SSPM interpixel surface.

Each of the 100 squares in Figure $4 \mathrm{~b}$ represents the spatial distribution of collection of photons emitted from that pixel. For instance, the lower-left square illustrates that photons originating from the lower-left pixel will be distributed evenly over the surface of the device and slightly less at the edges. In fact, this is the trend observed for all avalanche photons regardless of originating position.

When the above response matrix is summed, we arrive at the probability distributions for a photon originating at pixel $i$ and landing in any pixel. The approximate spatial uniformity of these distributions indicates that for either a diffusely-wrapped or specularly-wrapped crystal, any external optical crosstalk photon has an equal likelihood of arriving at any given pixel. Thus, a slightly modified uniform distribution might be used to determine avalanche photon fates in place of detailed Monte Carlo simulations. This external crosstalk mechanism is observed to contrast with the internal direct (ID) optical crosstalk mechanism which obeys a nearest neighbor or exponential decay type behavior. 


\section{Discussion}

For scintillation detection, the PDE of a SSPM can be improved by adding an aluminum fill layer over the interpixel region. The degree of improvement is higher for lower fill factor devices. External optical crosstalk will increase by a similar factor. The external optical crosstalk effect may be of little to no consequence if the SSPM is designed to be blue sensitive, because the hot carrier emissions peak at longer wavelengths. In addition, externally reflected avalanche photons must arrive at the SSPM surface with an angle great enough to overcome the crystal-interface barrier. This high angle will likely cause these photons to overshoot the high-multiplication region. This is in contrast to internal direct optical crosstalk in which avalanche photons may travel long distances laterally through the region of high multiplication. Optical crosstalk is not often a limiting factor, however it can be easily minimized by proper choice of operating bias. A simplified model for external optical crosstalk simulation will aid in the development of a complete stochastic model of the SSPM.

\section{References}

[1] A. L. Lacaita, F. Zappa, S. Bigliardi, and M. Manfredi, "On the bremsstrahlung origin of hot-carrier-induced photons in silicon devices," IEEE Trans. Electron Devices, vol. 40, no. 3, pp. 577-582, March 1993.

[2] F. Cayouette, D. Laurendeau, and C. Moisan, "DETECT2000: An Improved Monte-Carlo Simulator for the Computer Aided Design of Photon Sensing Devices," Proceedings of SPIE, vol. 4833, pp. 69-76, 2002.

[3] J. C. Jackson, D. Phelan, A. P. Morrison, R. M. Redfern, and A. Mathewson, "Towards integrated single photon counting microarrays," Opt. Eng., vol. 42, no. 1, pp. 112-118, January 2003.

[4] I. Rech, A. Ingargiola, R. Spinelli, I. Labanca, S. Marangoni, M. Ghioni, and S. Cova, "A New Approach to Optical Crosstalk Modeling in Single-Photon Avalanche Diodes," IEEE Photonics Tech. Letters, vol. 20, no. 5, pp. 330-2, March 2008.

[5] N. Akil, S. E. Kerns, D. V. Kerns, Jr., A. Hoffmann, and J. Charles, "A multimechanism model for photon generation by silicon junctions in avalanche breakdown," IEEE Trans. Electron Devices, vol. 46, no. 5, pp. 1022-1028, May 1999. 